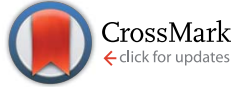

Cite this: RSC Adv., 2017, 7, 6474

Received 18th November 2016

Accepted 6th January 2017

DOI: 10.1039/c6ra27021b

www.rsc.org/advances

\section{Healing of skin wounds using a new cocoon scaffold loaded with platelet-rich or platelet-poor plasma $\uparrow$}

\author{
Jiawei Liu, $\stackrel{t}{+}^{\mathrm{a}}$ Fei Lu,,$_{+}^{\mathrm{ab}}$ HongLei Chen, ${ }^{\mathrm{a}}$ Rong Bao, ${ }^{\mathrm{c}}$ Zhiquan Li, ${ }^{\mathrm{c}}$ Bitao Lu, ${ }^{\mathrm{a}}$ Kun Yu, ${ }^{\mathrm{a}}$ \\ Fangying Dai, ${ }^{a b}$ Dayang $\mathrm{Wu}^{\mathrm{ab}}$ and Guangqian Lan ${ }^{\star a b}$
}

\begin{abstract}
The cocoons of the silkworm Bombyx mori are widely used as biofunctional materials. In the present study, cocoon composites (CCs) were treated with a solution consisting of calcium chloride, ethanol, and distilled water, resulting in partial dissolution, followed by freeze-drying, yielding cocoon scaffolds (CCSs). The CCSs were then immersed in autologous platelet-rich plasma (PRP) or platelet-poor plasma (PPP) obtained from rabbits and freeze-dried again, allowing us to harvest the other two composite materials (CCSs + PRP and CCSs + PPP). Analysis of the physical and biological properties of the materials demonstrated that the water absorption and retention capacities of CCSs had improved. After loading with PRP or PPP, both of the composite materials significantly promoted the growth of L929 cells, with CCSS + PRP showing slightly better results. Additionally, in vivo studies showed that both of the composite materials could enhance the process of wound healing, with the CCSs + PRP exhibiting the best results. Thus, our results suggested that these composites may have potential applications in the biomedical field.
\end{abstract}

\section{Introduction}

Skin is the largest organ in the human body and functions mainly to protect the body, release sweat, and mitigate feelings of cold, heat, and pressure. The skin covers the whole body and can prevent tissues and organs of the body from physical, mechanical, chemical, and pathogenic damage. ${ }^{\mathbf{1 , 2}}$ The skin is composed of the epidermis, dermis, and subcutaneous tissue, and damage to the skin causes the injured area to launch a series of subtle and complex mechanisms to rebuild its integrity and restore normal functions. ${ }^{3-5}$ Moreover, the wound healing process, which involves coagulation, inflammation, granulation tissue formation, epithelization, and tissue reconstruction, ${ }^{6,7}$ is affected by many factors, such as advanced age, malnutrition, and disease. ${ }^{7-9}$ Recent developments in tissue engineering and regenerative medicine have contributed to the development of tissue engineering skin substitutes; however, currently available skin substitutes still have several limitations, including the risk of rejection and

${ }^{a}$ College of Textile and Garments, Southwest University, Chongqing 400715, China. E-mail: 30353930@qq.com; Tel: +8613594005200

${ }^{b}$ Chongqing Engineering Research Center of Biomaterial Fiber and Modern Textile, Chongqing 400715, China

'The Ninth People's Hospital of Chongqing, Chongqing 400715, China

$\dagger$ Electronic supplementary information (ESI) available. See DOI: $10.1039 / \mathrm{c} 6 \mathrm{ra} 27021 \mathrm{~b}$

¥ These authors contributed equally to this study. infection. An ideal wound dressing should satisfy some conditions such as good biocompatibility, maintenance of a humid environment to prevent the wound from dehydrating, the capacity for blocking dust and bacteria from entering the wound, good permeability, and the ability to promote the efficient formation of epithelial tissue.

Silk consists of fibroin and sericin, and silk fibroin (SF) is a type of natural polymer fiber protein extracted from silk that has been widely used in biomedical purposes owing to its unique physical, mechanical, and biological properties, such as good elasticity, low molecular weight, good biocompatibility, and lack of pro-inflammatory effects. ${ }^{\mathbf{1 0}, 11} \mathrm{SF}$ contains peptides and can promote the differentiation and proliferation of human skin fibroblasts. ${ }^{12}$ Moreover, SF can accelerate wound healing when used as a skin wound dressing. ${ }^{\mathbf{1 3 , 1 4}}$ Sericin wraps around $\mathrm{SF}$ and plays a role in the protection and connection of SF, thus facilitating the formation of cocoons. ${ }^{15}$ Sericin may also have many biological properties and pharmacological properties such as anticancer activities, ${ }^{\mathbf{1 6}}$ promotion of digestion, ${ }^{\mathbf{1 7}}$ and inhibition of tyrosinase activity.$^{18}$ Moreover, Li et al. ${ }^{19}$ fabricated a three-dimensional poly(epsilon-caprolactone)/sericin porous nanofibrous scaffold by electrospinning and found that the addition of sericin could improve the hydrophilic capability. Additionally, the nanofibrous scaffolds could improve the adhesion and proliferation of human skin fibroblasts. Although all of these materials improved wound healing, they have several drawbacks, including complex preparation protocols and single structures. Furthermore, extraction of SF from 
natural cocoons can disrupt the structure of the cocoons to some extent. Natural cocoons are known to show good permeability and may have several benefits. For example, the good permeability will prevent the area surrounding the wound from becoming damp, which would promote wound healing. Second, stimulation with air would promote the coagulation of platelets, accelerating wound scabbing and thus promoting wound healing. Finally, the good permeability could inhibit the proliferation of anaerobic bacteria, which would prevent bacterial infection. Therefore, cocoons may be useful for the development of new wound dressings while maintaining the integrity of the natural structure.

Platelet-rich plasma (PRP) is a type of plasma containing highly concentrated platelets, produced by gradient density centrifugation of whole blood. After activation, PRP can release numerous growth factors such as basic fibroblast growth factor (bFGF), vascular endothelial growth factor (VEGF), epidermal growth factor (EGF), which play an important roles in the wound healing process..$^{\mathbf{2 0 2}}$ Some reports have demonstrated that PRP can promote cell proliferation and accelerate the process of wound healing. ${ }^{22,23}$ Carter et al. ${ }^{24}$ concluded that PRP could not only accelerate wound healing but also reduce the risk of serious wound infections. To date, many recombinant human growth factors have been produced and applied in the clinical setting. However, the high sensitivity and short halflives of these factors have limited their applicability and made their bioactivities unstable. ${ }^{25,26}$ This problem may be overcome by combining PRP with porous materials, allowing for the sustained release of components and therefore prolonging the active time of the material.

Accordingly, in this study, we used a ternary solution consisting of calcium chloride, alcohol, and distilled water to modify natural cocoons and obtain cocoon scaffolds (CCSs) with pores. Additionally, we combined the CCSs with PRP/ platelet-poor plasma (PPP) and evaluated the effects of this combination on the growth of L929 cells in vitro and on wound healing in rabbits in vivo. As illustrated in Fig. 1, the cocoon composites (CCs) were prepared with a two-step approach, including the preparation of CCSs and the preparation of autologous PRP and PPP. The CCSs could act as storage units for growth factors (bFGF, VEGF, and EGF) contained in PRP and PPP. Thus, these CCSs could have biomedical applications.

\section{Materials and methods}

\subsection{Animals and reagents}

Cocoons were obtained from Sichuan Antai Cocoon Group Co., Ltd. (Sichuan, China). Dulbecco's modified Eagle's medium (DMEM) was purchased from Gibco. Mepitel was obtained from Molnlycke Health Care Co. (Sweden). New Zealand white rabbits were obtained from the Animal Laboratory Center of the Third Military Medical University at 2 months of age. All rabbits were acclimated to the laboratory environment for at least 1 week before use in experiments. The animals were kept in metal cages and maintained under controlled conditions at a temperature of $21-25{ }^{\circ} \mathrm{C}$ with a relative humidity of $45-65 \%$ and a 12 h light-dark cycle. L929 murine fibroblasts (ACTT, CCL-1) were obtained from Chongqing University of Technology. All other chemicals used in this study were of analytical grade.

\subsection{Preparation of cocoon dressings}

For preparation of CCSs, a solution was prepared by mixing calcium chloride, ethanol, and deionized water (molar mass ratio, $1: 2: 8$ ). Briefly, calcium chloride was added to deionized water, and the mixture was stirred until the salt was completely dissolved. Ethanol was then added, and the solution was allowed to cool to room temperature naturally. Both ends of the cocoons were cut off, and silkworm pupae were discarded and washed twice to remove impurities on the surface of the cocoons. The cocoons were then moved to glass bottles with the solution and incubated in a $58{ }^{\circ} \mathrm{C}$ water bath for $1 \mathrm{~h}$. Finally, the cocoons were removed, washed again, and freeze-dried to obtain CCSs. The CCSs were stored with desiccant in polyethylene bags if not used immediately.

For preparation of autologous PRP and PPP, $10 \mathrm{~mL}$ blood was obtained from the hearts of New Zealand white rabbits and then centrifuged in tubes containing $1 \mathrm{~mL}$ of $3.8 \%$ sodium citrate as an anticoagulant. PRP was obtained via a two-step centrifugation method. The blood was first centrifuged at 400 $\times g$ for $15 \mathrm{~min}$ and then recentrifuged at $800 \times g$ for $15 \mathrm{~min}$; the clear supernatant phase was considered PPP, whereas the other phase was harvested as PRP.

For preparation of cocoon dressings loaded with PRP and PPP, the PRP and PPP were activated by adding $0.05 \mathrm{~mL}$ of $10 \%$

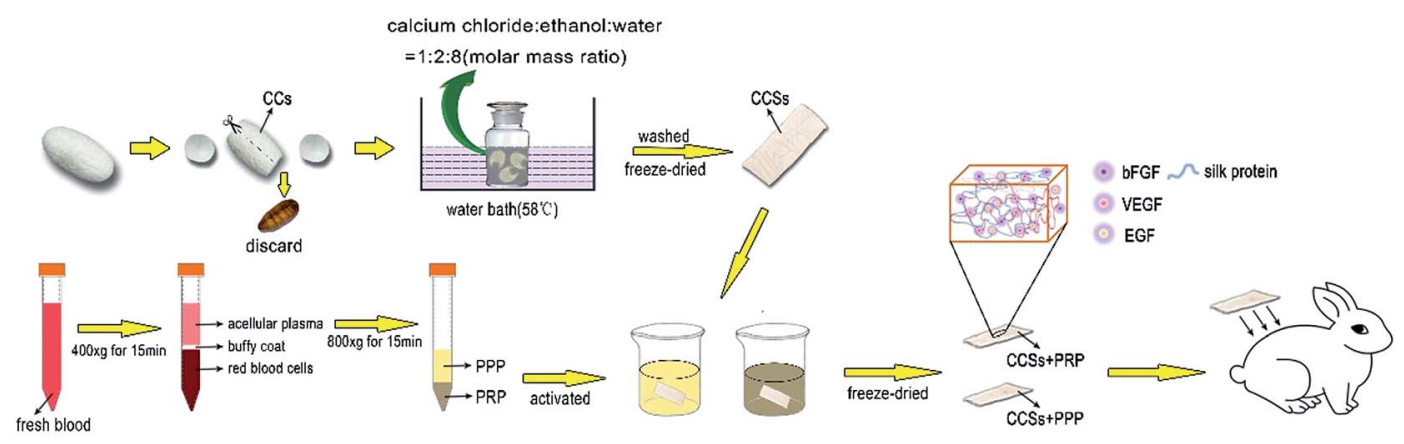

Fig. 1 The process used for application of cocoon composites as a wound dressing. 
calcium chloride solution per $1 \mathrm{~mL}$ PRP or PPP, and CCSs were then prepared before immersion in PRP or PPP for $30 \mathrm{~min}$, followed by freezing at $-20{ }^{\circ} \mathrm{C}$ for $12 \mathrm{~h}$. The samples were then lyophilized at $-50{ }^{\circ} \mathrm{C}$ for $24 \mathrm{~h}$ in a freeze drying machine.

\subsection{Characterization of the samples by scanning electron microscopy (SEM)}

SEM (S600; Hitachi, Japan) was used to characterize the morphology and arrangement of the samples. Prior to imaging, the samples were mounted on aluminum stubs and coated in gold for better conductivity.

\subsection{Characterization of the mechanical properties of the samples}

The mechanical properties of the samples were measured using a universal material testing instrument (AGS-X, Shanghai, China). ${ }^{27}$ Samples were prepared by freeze-drying; wet samples were prepared by immersion of the dry samples in water for $10 \mathrm{~min}$, and both dry and wet samples were subjected to tensile tests at a constant rate of $20 \mathrm{~mm} \mathrm{~min}^{-1}$. The same sample was tested five times.

\subsection{Characterization of porosity}

The porosity of the cocoon was determined using the liquid displacement method. ${ }^{28}$ Sample dimensions were measured using vernier calipers to calculate the volume, and then sample was placed in an oven at $45^{\circ} \mathrm{C}$ for $24 \mathrm{~h}$, weighed, and soaked in a known volume of ethanol for $24 \mathrm{~h}$ at ambient temperature to allow the ethanol to penetrate into the pores of the sample. The test specimens were then removed and hung to dry under gravity on a rope for $30 \mathrm{~s}$, after which the samples were weighed immediately to determine the final weight. The same sample was tested five times. The porosities of the samples were calculated using the following formula:

$$
\operatorname{Porosity}(\%)=\left(\frac{\left(W_{\mathrm{w}}-W_{\mathrm{d}}\right)}{\rho_{\text {ethanol }}} \times V\right) \times 100 \%
$$

where $W_{\mathrm{w}}$ and $W_{\mathrm{d}}$ represent the weights before and after contact with ethanol, respectively, and $\rho_{\text {ethanol }}$ and $V$ represent the density of ethanol and the volume of the sample, respectively.

\subsection{Characterization of the physical properties of the samples}

The crystallinities of the samples were detected by X-ray diffraction (XRD; D/MAX-2550; Rigaku, Japan). The samples were cut into the powder form before testing. The hydrophilicities of samples were analyzed by measuring the contact angles (OCA15EC; Dataphysics, Germany).

The water absorption and retention capacities of samples were evaluated using the gravimetric method. For water absorption tests, samples were immersed in water at $37{ }^{\circ} \mathrm{C}$ and then taken out and weighed at different times. The swelling ratio was determined using the following formula:

$$
\text { Swelling ratio }(\%)=\left(\frac{W_{\mathrm{a}}-W_{\mathrm{b}}}{W_{\mathrm{b}}}\right) \times 100 \%
$$

where $W_{\mathrm{a}}$ and $W_{\mathrm{b}}$ represent the weights of wet and dry samples, respectively.

For water retention tests, samples (water saturated) were incubated at $37{ }^{\circ} \mathrm{C}$ and weighed at each time point. The water retention ratio was determined using the equation:

$$
\text { Water retention ratio }(\%)=\left(\frac{W_{\mathrm{c}}-W_{\mathrm{d}}}{W_{\mathrm{c}}}\right) \times 100 \%
$$

where $W_{\mathrm{c}}$ and $W_{\mathrm{d}}$ represent the weights of wet and dry samples, respectively.

\subsection{Release of growth factors from the composite dressings}

The release of growth factors was carried out under static conditions. The release speed of the growth factors was measured using normal saline (NS), phosphate-buffered saline (PBS), and simulated body fluid (SBF). The SBF was prepared as described in the literature. ${ }^{29}$ Each lyophilized sample $(\sim 0.05 \mathrm{~g})$ was soaked in $8 \mathrm{~mL}$ NS, PBS, or SBF at $37{ }^{\circ} \mathrm{C}$, and soaked solutions were obtained within a certain time for the quantification of growth factors released by the samples. The glasses were sealed with plastic wrap to prevent the loss of moisture. The concentrations of bFGF, VEGF, and EGF were quantified using enzyme-linked immunosorbent assays (ELISAs; R\&D Systems, Shanghai, China).

\subsection{Cytotoxicity studies in vitro}

L929 cells were used to evaluate the cytotoxicity in vitro. Briefly, cells were cultured in DMEM containing 10\% fetal bovine serum (FBS) and $3 \%$ antibiotics $\left(200 \mathrm{mg} \mathrm{mL}^{-1}\right.$ penicillin and $200 \mathrm{mg} \mathrm{mL}^{-1}$ streptomycin) in a $\mathrm{CO}_{2}$ incubator for $12-24 \mathrm{~h}$ until attached. The culture fluid was then replaced with fresh DMEM and cultured for another 24-48 h. Next, the cell culture fluid was discarded, and cells were detached by incubation in $1 \mathrm{~mL}$ trypsin-EDTA solution (0.25\% trypsin and $0.02 \%$ EDTA) for $1 \mathrm{~min}$, followed by addition of $3 \mathrm{~mL}$ culture medium to terminate digestion. Cells were harvested and centrifuged at $1000 \mathrm{rpm}$ for $5 \mathrm{~min}$. The supernatant was discarded, and the cell pellet was diluted with culture medium. Cells were cultured again at $37{ }^{\circ} \mathrm{C}$ in an atmosphere containing $5 \% \mathrm{CO}_{2}$ for 24,48 , or $96 \mathrm{~h}$. Finally, the cells were immersed in tissue fixative $(10 \%$ neutral-buffered formalin) for $24 \mathrm{~h}$ for immobilization and observed by SEM. The L929 cells $\left(1 \times 10^{4}\right.$ cells per well $)$ were incubated with samples (diameter: $5 \mathrm{~mm}$ ) in 96-well plates at $37{ }^{\circ} \mathrm{C}$ for $24 \mathrm{~h}$ and then observed and photographed using a fluorescence microscope (Nikon). The viability of the cells was measured using a Calcein-AM/PI Double Stain Kit (40747ES76; Yeasen Biological Technology Co., Ltd, Shanghai, China) following the manufacturer's instructions.

3-(4,5-Dimethyldiazol-2-yl)-2,5-diphenyl tetrazolium bromide (MTT) assays were used to evaluate negative effects of the materials on the cells. ${ }^{30}$ Briefly, lyophilized samples were cut into small wafers (diameter: $5 \mathrm{~mm}$ ) and transferred into 96-well plates. Cell suspensions $\left(1 \times 10^{4}\right.$ cells per well $)$ were then seeded, and plates were incubated for 24,48 , or $72 \mathrm{~h}$. For MTT assays, 10 $\mu \mathrm{L}$ MTT solution was added to each well, and cells were cultured for another $4 \mathrm{~h}$. Subsequently, the formazan reaction product was 
dissolved by the addition of $100 \mu \mathrm{L}$ dimethylsulfoxide (DMSO). The absorbance was measured on a microplate reader (Multiskan MK3) at $490 \mathrm{~nm}$. The data were obtained by averaging the measured values of five replicates.

\subsection{Surgical procedure}

New Zealand white rabbits were used to evaluate the wound healing characteristics of samples. All experiments were approved by the Animal Ethics Committee of our institution. All animal experiments and care were approved by the National Center of Animal Science Experimental Teaching (ASET) at the College of Animal Science and Technology (CAST) in the Southwest University of China and were in accordance with the "Guide for the Care and Use of Laboratory Animals". The rabbits were anesthetized by intramuscular injection of xylazine hydrochloride at a dose of $0.2 \mathrm{~mL} \mathrm{~kg}^{-1}$, and the limbs were fixed on a rabbit anatomy set. The hair was shaved off the backs of the rabbits, and the skin was disinfected with $75 \%$ alcohol. Fullthickness skin wounds measuring $2 \mathrm{~cm}$ in diameter were created on the dorsum of the rabbits. The first wound acted as a control without any treatment, whereas the other wounds were covered with CCSs, CCSs + PRP, CCSs + PPP, or Mepitel. All wounds were covered with sterilized gauze and fixed with an elastic bandage. After surgery, the rabbits were euthanized on days 5,10 , or 15 . The wounds were photographed, and the percent reduction in wound size was calculated using the following formula:

$$
\text { Wound size reduction }(\%)=\left(\frac{A_{\mathrm{i}}-A_{t}}{A_{\mathrm{i}}}\right) \times 100 \%
$$

where $A_{\mathrm{i}}$ represent the area of the initial wound, and $A_{t}$ represents the wound area at the time the samples were obtained.

\subsection{Histological evaluation}

For histological analysis, the tissue specimens were harvested by excision of the skin (containing the entire wound and adjacent normal skin), fixed in $10 \%$ buffered formalin for $24 \mathrm{~h}$, embedded in paraffin, and sectioned to 3-5 $\mu \mathrm{m}$ thickness. Sections were stained with hematoxylin and eosin (H\&E) and Masson's trichrome staining for detection of collagen fibers. The sections were observed under a DXM 1200F microscope (Nikon H600L; Germany).

\subsection{Standard analysis}

All results were statistically analyzed using Student's $t$-tests or one-way analysis of variance (ANOVA). Results with $P$ values of less than were considered statistically significant. All results were expressed as means \pm standard deviations (SDs).

\section{Results}

\subsection{Characterization of CCSs and CCs}

The microscopic surface of materials is shown in Fig. 2. The CCs exhibited a dense structure, whereas the CCSs showed a well interconnected porous distribution. Moreover, with the addition of PRP or PPP to the CCSs, a layer of membrane was observed; this layer appeared to consist of platelets and some proteins included in PRP and PPP. The interlinking porous structure within a scaffold could contribute to the attachment and proliferation of cells. ${ }^{31}$ Thus, CCSs + PRP and CCSs + PPP may have applications as wound dressings owing to the dissolution of sericin and the decreased adhesion between the silk, resulting in the formation of a porous structure after freezedrying.

XRD patterns of CCs, CCSs, CCSs + PRP, and CCSs + PPP scanned within $10-70^{\circ}$ at position $2 \theta$ are shown in Fig. 3A. The peaks present around $20^{\circ}$ were decreased compared with that of CCs, demonstrating that the crystallinity was reduced after treatment.

The porous structures of CCs, CCSs, CCSs + PRP, and CCSs + PPP were evaluated. The cocoons showed $0.98 \%$ porosity, whereas the others showed porosities of $84.2 \%, 82.7 \%$, and $83.1 \%$, respectively (Fig. 3B). The significant increase in porosity could be beneficial to the hydrophilicity of materials and could enhance water content.

Good mechanical properties are essential for wound dressings. Thus, we determined the tensile properties of CCs, CCSs, CCSs + PRP, and CCSs + PPP (Table 1). The tensile strength of dry CCS was about $0.58 \pm 0.31 \mathrm{MPa}$, and the elongation at the break point was $9.53 \% \pm 1.26 \%$; these values were decreased by approximately $94.7 \%$ and $37.3 \%$, respectively, compared with those if CCs. Interestingly, in the wet state, the elongation at the break point of wet CCSs increased to $102.43 \% \pm 18.72 \%$, which was about 10 fold higher than that in the dry state. However, the reduction in tensile strength was acceptable because the CCs were too hard and not suitable for wound dressings. In contrast, although the tensile strength was greatly reduced, the wet CCSs were soft and would not cause secondary damage to the wound. Additionally, the intensity of CCSs was sufficient for use as a wound dressing. Furthermore, the addition of PRP and PPP do not have much effect on the mechanical properties of the scaffolds.

Ideal wound dressing must possess good hydrophilicity to maintain the moistness of the wound and prevent dehydration. The surface wettability of the biomaterial will affect the adsorption of proteins and the migration, adhesion, and proliferation of cells. ${ }^{32-34}$ As shown in Fig. 4A, the water droplet still remained intact on the surface of CCs after $60 \mathrm{~s}$, demonstrating poor water wettability (contact angle $\theta>90^{\circ}$ ), whereas the other three groups show good hydrophilicity, with the water droplet almost completely absorbing into the materials within $6 \mathrm{~s}$ (contact angle $\theta<90^{\circ}$ ). The swelling and water retention ratios of samples are shown in Fig. $4 \mathrm{~B}$ and $\mathrm{C}$. The results were consistent with the contact angle tests. Specifically, the swelling ratio of the CCSs was about 10 times its dry weight, while that of the CCs was only 1.4 times its dry weight. Moreover, the CCSs could retain water for a longer time, and the CCSs + PRP and CCSs + PPP samples showed similar trends in hydrophilic capacity.

\subsection{Release rates of growth factors from CCs}

Next, the release rates of growth factors (EGF, VEGF, and bFGF) from CCs were investigated (Fig. 5). Both CCSs + PRP and CCSs 

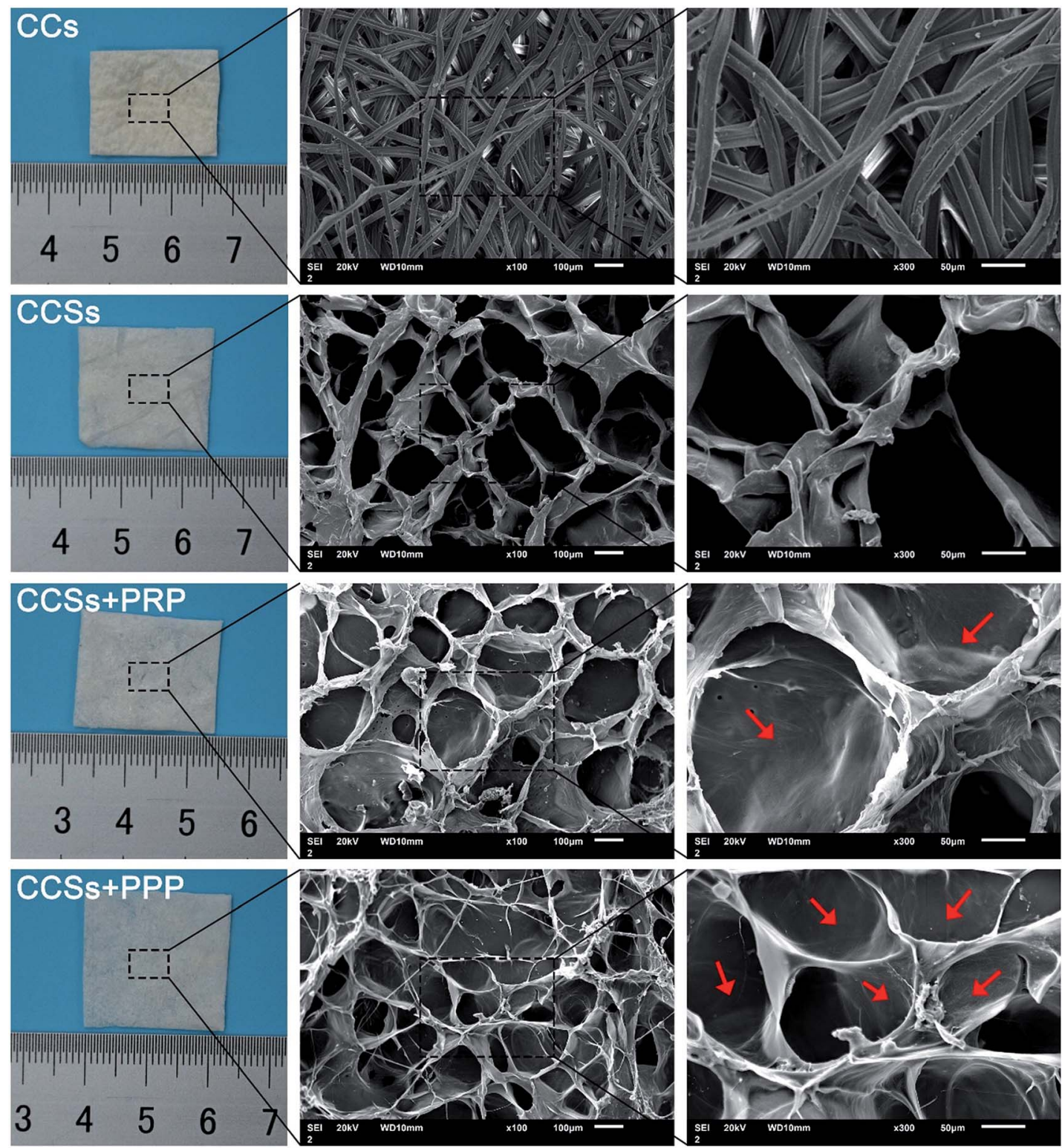

Fig. 2 SEM images of CCs, CCSs, CCSs + PRP, and CCSs + PPP
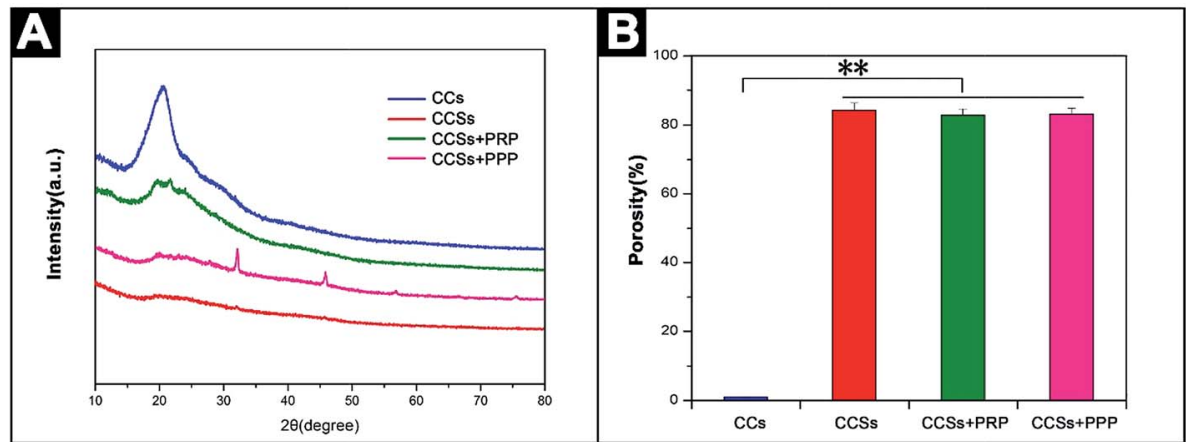

Fig. 3 (A) XRD analysis of CCs, CCSs, CCSs + PRP, and CCSs + PPP. (B) Porosity studies of CCs, CCSs, CCSs + PRP, and CCSs + PPP. **P<0.01, $n=5$. 
Table 1 Tensile properties of CCs, CCSs, CCSs + PRP, and CCSs + PPP

\begin{tabular}{|c|c|c|c|c|}
\hline Materials & & Tensile strength (MPa) & Young's modulus (MPa) & Elongation at break (100\%) \\
\hline \multirow[t]{2}{*}{ CC } & Dry & $22.18 \pm 1.52$ & $80.86 \pm 2.17$ & $15.21 \pm 1.74$ \\
\hline & Wet & $24.46 \pm 1.68$ & $76.95 \pm 2.37$ & $14.78 \pm 1.86$ \\
\hline \multirow[t]{2}{*}{ CCS } & Dry & $0.58 \pm 0.31$ & $17.27 \pm 1.27$ & $9.53 \pm 1.26$ \\
\hline & Wet & $0.34 \pm 0.16$ & $8.42 \pm 0.33$ & $102.43 \pm 18.72$ \\
\hline \multirow[t]{2}{*}{ CCS + PRP } & Dry & $0.48 \pm 0.13$ & $18.78 \pm 1.17$ & $10.44 \pm 1.47$ \\
\hline & Wet & $0.29 \pm 0.09$ & $7.57 \pm 0.57$ & $124.36 \pm 14.26$ \\
\hline \multirow[t]{2}{*}{$\mathrm{CCS}+\mathrm{PPP}$} & Dry & $0.44 \pm 0.25$ & $20.49 \pm 1.11$ & $8.92 \pm 1.13$ \\
\hline & Wet & $0.31 \pm 0.22$ & $9.43 \pm 0.45$ & $113.29 \pm 12.92$ \\
\hline
\end{tabular}

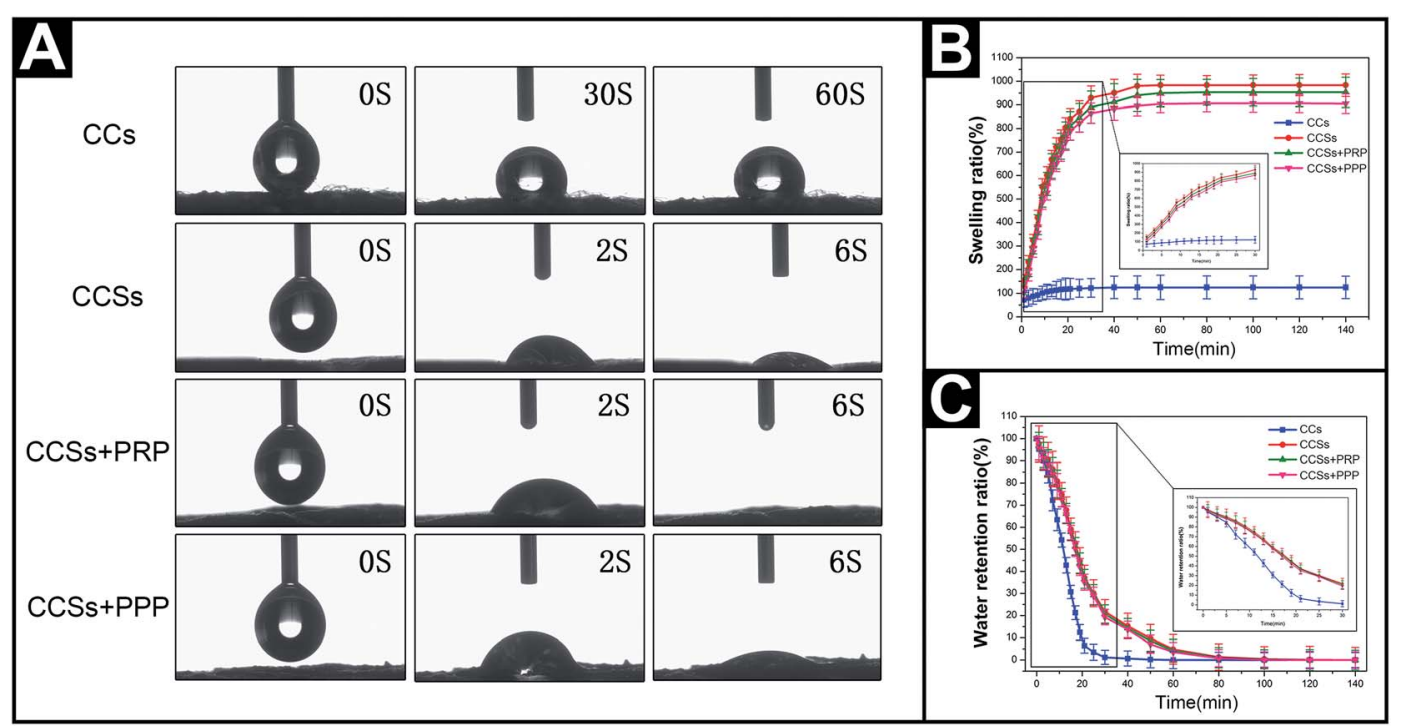

Fig. 4 The hydrophilicities of CCs, CCSs, CCSs + PRP, and CCSs + PPP. (A) Contact angle, (B) swelling ratio, and (C) water retention ratio.

+ PPP could release all three growth factors in NS. As the immersion time increase from 0.5 to $72 \mathrm{~h}$ (Fig. $5 \mathrm{~A}$ ), the amount of EGF in the CCSs + PRP group increased from 30.7 to $350.1 \mathrm{pg}$ $\mathrm{mL}^{-1}$, which was 2-3 times greater than that of the CCSs + PPP group during the same time; VEGF and bFGF showed the same trends, and similar trends were observed in PBS (Fig. 5B) and SBF (Fig. 5C). The release profiles could be divided into three parts: a burst release $\mathrm{e}^{35,36}$ in the initial $24 \mathrm{~h}$, a stable and regularly release from 24 to $72 \mathrm{~h}$, and a stagnant release for the remaining time (Fig. S1 $\dagger$ ). During the first stage in the NS group, the amount of EGF was increased from 30.7 to $280.5 \mathrm{pg} \mathrm{mL}^{-1}$, which was about 9-fold higher than the initial concentration at $24 \mathrm{~h}$. However, during the second stage, the amount of EGF was increased from 280.5 to $304.1 \mathrm{pg} \mathrm{mL} \mathrm{m}^{-1}$. The increase was stable and regular throughout the $48 \mathrm{~h}$ period. During the third stage, the amount of EGF no longer increased. The other group exhibited a similar trend. These data indicated that PRP and PPP combined with CCSs successfully, and the CCSs could be regarded as a storage vehicle for growth factors, which could be released from the interconnected porous structure of CCSs. Additionally, growth factors almost could not be detected from CCs loaded with PRP or PPP, indicating that CCs could not act as storage vehicles for growth factors (Fig. S2†). The release of a compound from a matrix was controlled by several factors, including the hydrophilic nature ${ }^{37}$ and pore size of the scaffold $;^{38}$ higher pore sizes will result in releasing greater amounts of compounds. Notably, ELISA measurements offer a measurement of the quantity of growth factors released by the composites under certain circumstances (NS, PBS, and SBF). Thus, the actual release of growth factors may be slightly different when the composites are used as a wound dressing in vivo. Accordingly, we next evaluated the biological properties of the materials in vitro and in vivo.

\subsection{In vitro studies}

To investigate the biological functions of the materials, we added the materials directly in cultures of L929 cells, as shown in the schematic sketch in Fig. 6. MTT assays in L929 cells treated with the materials are shown in Fig. 6A. All measurements of absorbance measured exceeded $90 \%$, demonstrating that all samples had low toxicity. CCSs with higher pore distributions exhibited higher cell viabilities than CCs after $24 \mathrm{~h}$ incubation. After loading with PRP and PPP, cell viabilities were significantly increased, reaching $154.8 \%$ and $133.7 \%$, respectively. The same trends were observed after 48 or $72 \mathrm{~h}$ of 


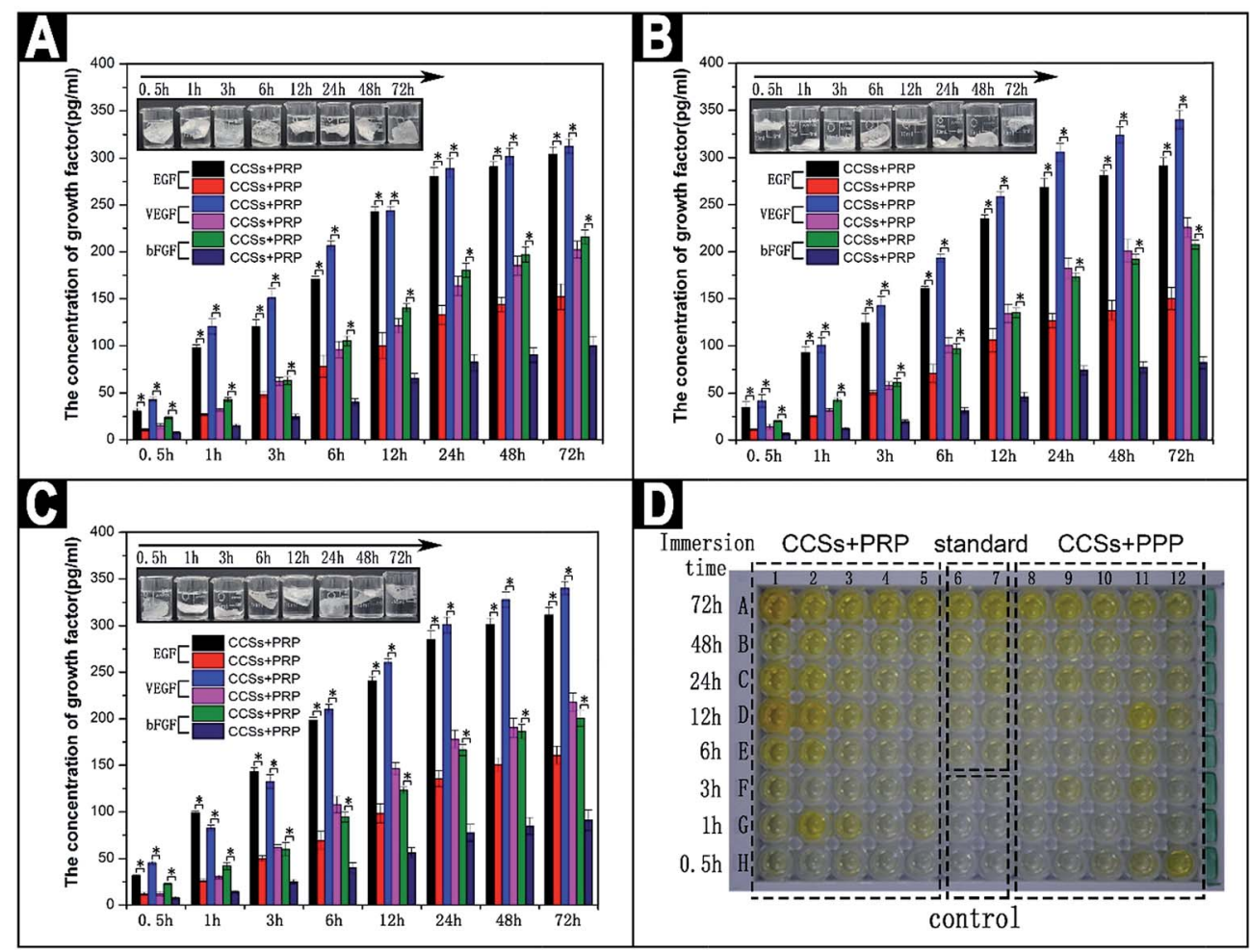

Fig. 5 Release rates of growth factors from CCSs + PRP and CCSs + PPP in (A) NS, (B) PBS, and (C) SBF. The concentrations of growth factors in soaking liquid were measured at $0.5,1,3,6,12,24,48$, and $72 \mathrm{~h}$. (D) Addition of samples to 96 -well plates. $* P<0.05, n=5$.

incubation. In the presence of CCs, the growth of L929 cells was slightly poorer (Fig. 6Ba and b) compared with that of L929 cells treated with basic medium. In contrast, the growth of L929 cells treated with CCSs, CCSs + PRP, and CCSs + PPP exhibited different degrees of increased cell proliferation (Fig. 6Bc-e), and the PRP group exhibited the best performance in cell proliferation. The results of live/dead cell staining are shown in Fig. 6C. Notably, both CCSs + PRP and CCSs + PPP significantly promoted cell growth compared with those in the other groups; the amount of live cells (green) in the CCSs + PRP and CCSs + PPP groups was higher than those in the other three groups, particularly in the CCSs + PRP group, in which cell growth was the highest. Moreover, all groups showed different degrees of cell death (red), and CCSs + PRP showed the best performance.

\subsection{In vivo studies}

To demonstrate the practical applicability of the composite materials, we performed in vivo experiments to evaluate the effects of the materials on wound healing. Fig. 7A shows the photographs $(2 \mathrm{~cm} \times 2 \mathrm{~cm})$ of postoperative wound healing after treatment with CCSs, CCSs + PRP, CCSs + PPP, or Mepitel for 5,10 , and 15 days. On day 5 , all wounds exhibited calluses. After 10 days of treatment, all wounds started to contract from the edges, and the wounds covered with CCSs + PRP and CCSs + PPP contracted more quickly and showed $82.6 \%$ and $67.4 \%$ decreases in wound size, respectively (Fig. 7B), thereby demonstrating effective healing. The day 15 post surgery, complete re-epithelialization of wounds treated with CCSs +
PRP was observed, while other groups failed to heal completely.

Histological analysis of the wounds was also performed by H\&E staining, as shown in Fig. 7C. On day 5, all groups showed increased inflammatory cells, while some fibroblasts and collagen fibers appeared on the wound treated with CCSs + PRP. For the CCSs + PPP, although the numbers of fibroblasts and collagen fibers were lower, this material showed better effects than the other three materials, indicating that both PRP and PPP could contribute to wound healing. On day 10, there were still some inflammatory cells remaining on the untreated wounds and wounds treated with CCSs and Mepitel; however, fewer inflammatory cells were observed on wounds treated with CCSs + PRP and CCSs + PPP. Additionally, the epidermis and dermis started to form in the PRP and PPP groups, and some microvessels were observed in the PRP and PPP groups. On day 15 , wounds treated with CCSs + PRP were covered by a complete and thickened epidermis, while those in the other groups were covered with an incomplete, thin epidermis.

Masson's trichrome staining was used to visualize the formation and distribution of collagen during the healing process (Fig. 7D). The collagen fibers could be stained green, and the intensity represented the relative collagen content in the tissue sections. On day 5 , when compared with the other wound site, the CCSs + PRP-treated group showed a higher density of collagen fibers and increased neovascularization. On day 10 , the formation of new collagen fibers and neovascularization were more prominent for wounds treated with 


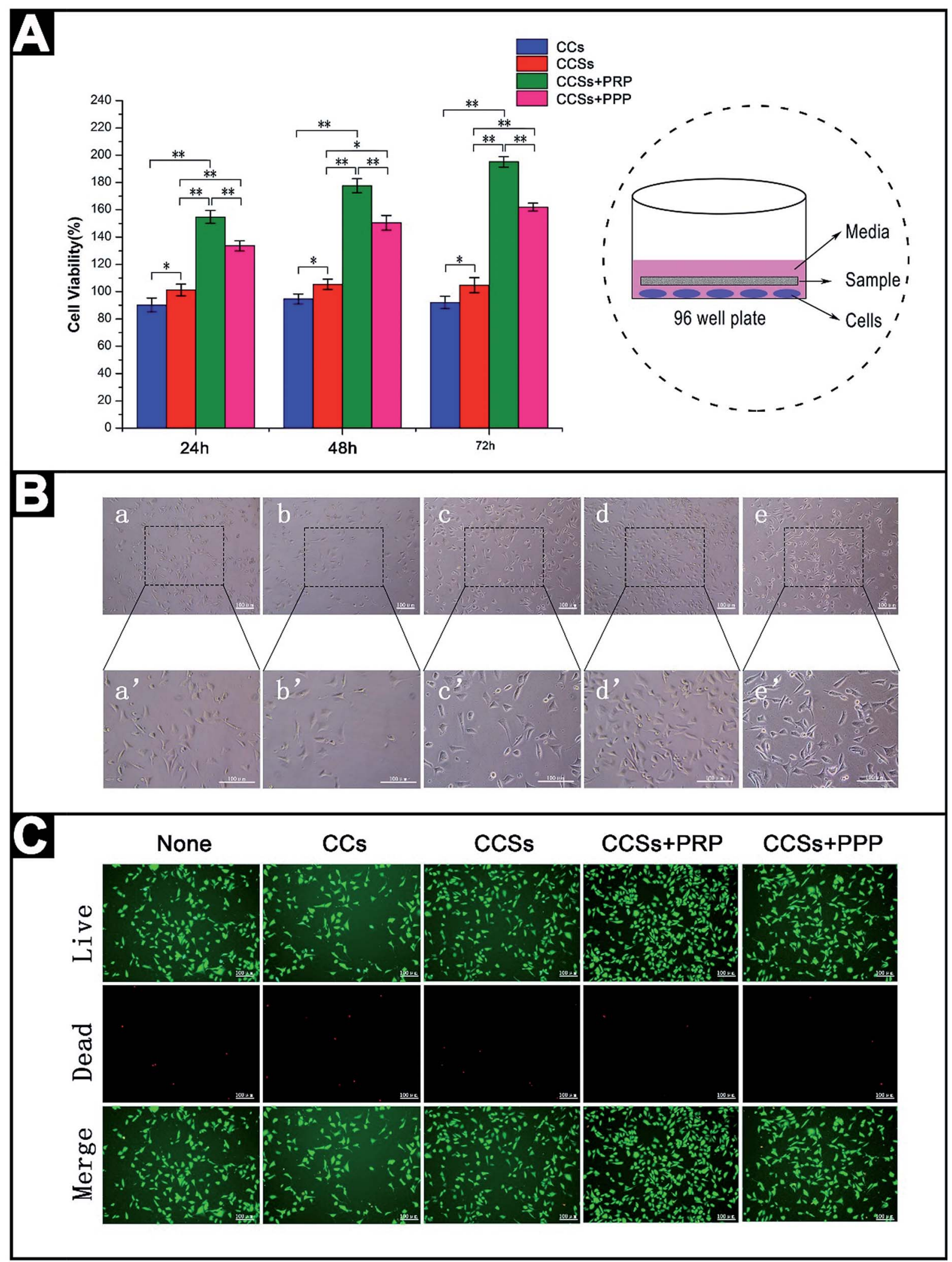

Fig. 6 (A) Cytotoxicity assays. L929 cells were incubated with CCs, CCSs, CCSs + PRP, or CCSs + PPP for 24, 48, or 72 h. The dotted circle shows a schematic of the culture of L929 cells in the presence of the materials. (B) Bright field microscopy images of L929 cells treated with basic DMEM $\left(a, a^{\prime}\right), C C\left(b, b^{\prime}\right), C C S\left(c, c^{\prime}\right), C C S+P R P\left(d, d^{\prime}\right)$, and CCS + PPP $\left(e, e^{\prime}\right)$ for $24 \mathrm{~h}$. (C) Fluorescence microscopy images of L929 cells treated with basic DMEM, CCs, CCSs, CCSs + PRP, and CCSs + PPP for $24 \mathrm{~h}$. Live cells were stained with calcein-AM dye and produced steady green fluorescence in live cells (excitation/emission at 495/515 nm, respectively). Dead cells were stained with propidium iodide (PI) and emitted bright red fluorescence in dead cells (excitation/emission at 495/635 nm, respectively). The scale bar represents $100 \mu \mathrm{m} . * P<0.05, * * P<0.01, n=5$. 


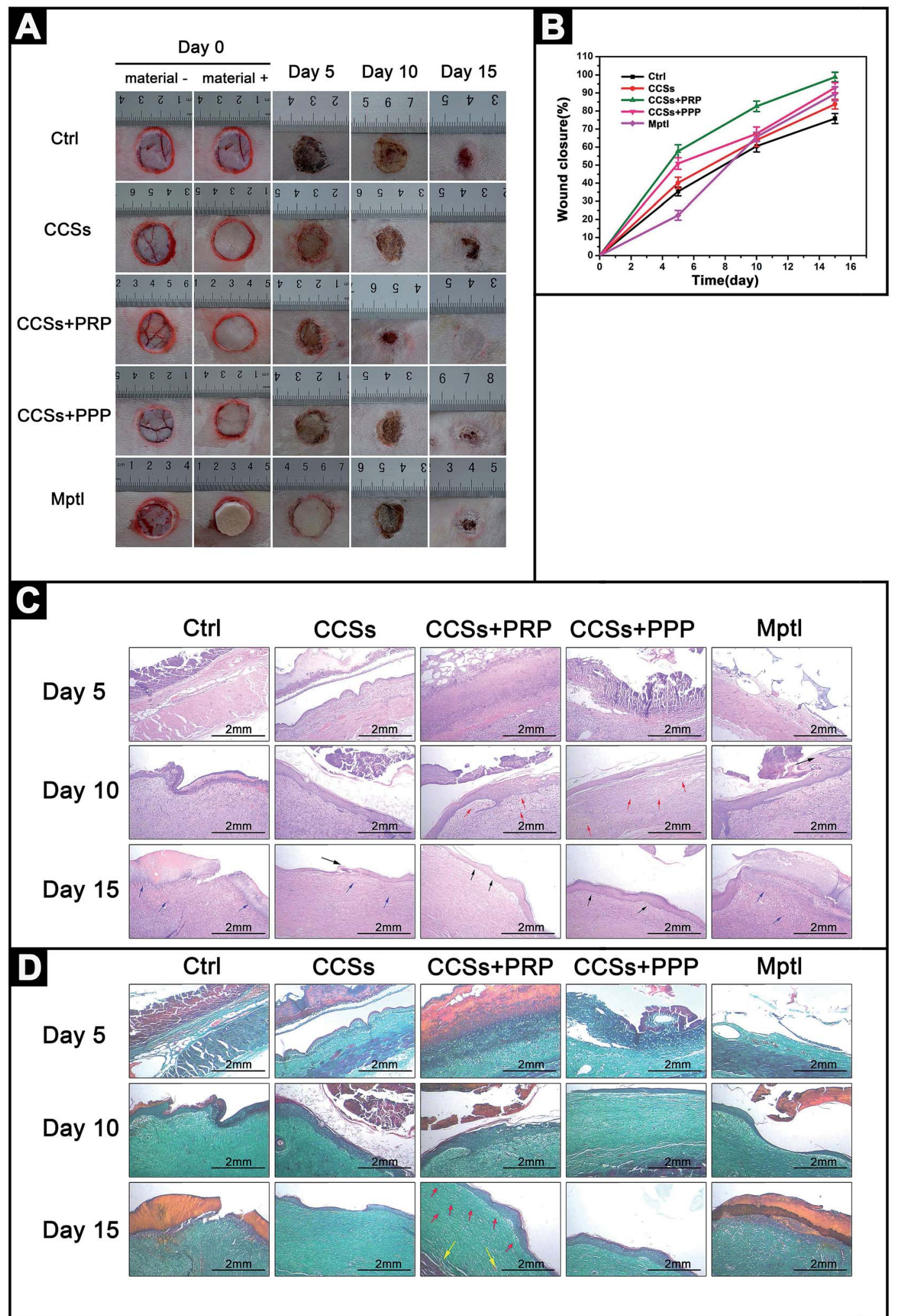

Fig. 7 (A) Visual observation of surface wound healing in rabbits. (B) Wound closure. Histological analysis of wound tissue using (C) hematoxylin and eosin staining. The red arrows represent fibroblasts, the black arrows represent the newly formed epidermis, and the blue arrows represent inflammatory cells. (D) Masson's trichrome staining. The red arrows represent collagen fibers, and the yellow arrows represent the nuclei.

CCSs + PRP, indicative of improved wound healing. However, significant collagen content was not observed in the other groups. On day 15, wounds treated with CCSs + PRP showed an identical distribution of collagen and blood vessels, while those in the other groups displayed less collagen and reduced blood vessel formation. General speaking, increased fibroblast 
formation and collagen secretion contributed to wound healing. Thus, our results indicated that CCSs + PRP was a promising biological material.

\section{Discussion}

In this study, XRD analysis showed that the crystallinity was reduced after treatment, which could be explained by dissolution of the sericin and weakening of the adhesion between silk, thus forming a pore structure with lyophilization. Accordingly, we concluded that the silks of CCSs, CCSs + PRP, and CCSs + PPP were in the amorphous form. Improvement of the hydrophilicity could be explained by improvement of the contact area between water and the materials in the highly interconnected porous structure, thus helping the solvent to flow into the scaffolds rapidly. Additionally, the capillary force could also help the scaffolds retain more solvent, and the mechanism of solvent uptake may involve diffusion..$^{39}$

In this study, CCSs were prepared successfully, and the features of this material were evaluated. The hydrophilicity was improved owing to the formation of a pore structure, which may be helpful for absorbing exudates and contributing to wound healing. Additionally, the mechanical properties of CCSs were reduced. However, the material remained elastic under wet conditions; the mechanisms controlling these effects are still unclear, and further studies are required.

Some studies have demonstrated that a single application of PRP is not sufficient for inducing the formation of new capillaries due to the short biological half-life of growth factors; ${ }^{40}$ however, repeated application of PRP may be sufficient. ${ }^{7}$ Repeated preparation of PRP is complicated. Accordingly, in this study, the CCSs + PRP were prepared in order to overcome the disadvantages of pure PRP. PRP is a combination of multiple growth factors, most of which play important roles in wound healing. The difference between PRP and PPP is that PPP contains many serum proteins but fewer platelets. The $\alpha$-granules of platelets will degranulate and release growth factors once platelets are activated. ${ }^{41}$ Moreover, some reports ${ }^{42,43}$ have demonstrated that freeze-dried platelets have effects similar to those of native platelets. Platelets are essential for the process of wound healing, ${ }^{\mathbf{4 4 , 4 5}}$ and studies have shown that platelet-derived growth factor also has important roles in wound healing. ${ }^{46,47}$ In this study, the CCSs were immersed in PRP or PPP and absorbed as much PRP or PPP as possible; after freeze-drying, the platelets were stored in CCSs. Thus, the CCSs could be regarded as a storage vehicle for growth factors, and when CCSs + PRP and CCSs + PPP are then used as wound dressings, the growth factors will be released from CCSs, thereby accelerating wound healing.

In this study, we used L929 cells used to evaluate the biocompatibilities of the materials. Our results indicated that the addition of PRP or PPP could significantly improve the growth of L929 cells and decrease L929 cell apoptosis. Moreover, growth factors may play a dominant role in this process. Both EGF and VEGF can stimulate mitogenesis in cells. ${ }^{48}$ Interestingly, porous structures are beneficial for the absorption of exudates, accumulation of oxygen, and retention of water, ${ }^{49}$ thus providing a suitable moisture environment for cell adsorption and proliferation..$^{31}$ The CCSs contained more pores and showed better performance in terms of cellular growth. Moreover, the addition of PRP and PPP could significantly promote the growth of L929 cells, mainly due to the presence of growth factors; indeed, both CCSs + PRP and CCSs + PPP could release growth factors gradually over the course of $72 \mathrm{~h}$. When the skin is damaged, the initial $72 \mathrm{~h}$ is an important period, and the release of growth factors during this period is expected to greatly improve wound healing. Additionally, in vivo studies showed that CCSs + PRP exhibited the best performance compare with the other groups, whereas the PPP group showed the second best performance, indicating that the concentration of platelets may be an important factor in wound healing. Notably, these findings were consistent with a previous study. ${ }^{50}$ Moreover, variations in platelets may be related to immune responses..$^{51}$ In our study, this problem was overcome by using autologous platelets from the rabbits themselves. Thus, our results showed that CCS + PRP was a good biological material that could facilitate wound healing.

\section{Conclusion}

In conclusion, CCSs exhibited decreased mechanical properties and become soft and hydrophilic compared with CCs, suggesting that CCSs may be more suitable for application as a wound dressing. In vitro studies showed that CCSs + PRP could significantly promote proliferation and decrease apoptosis in L929 cells. Moreover, in vivo animal studies showed that CCSs + PRP could accelerate wound healing more effectively. Thus, our findings indicated that this composite material was a promising biological material with applications in wound healing.

\section{Acknowledgements}

This work was supported by a grant from the Hi-Tech Research and Development 863 Program of China (grant no. 2013AA102507).

\section{References}

$1 \mathrm{H}$. P. Ehrlich and T. M. Krummel, Regulation of wound healing from a connective tissue perspective, Wound Repair Regen., 1996, 4, 203-210.

2 P. Chandika, S. C. Ko and W. K. Jung, Marine-derived biological macromolecule-based biomaterials for wound healing and skin tissue regeneration, Int. J. Biol. Macromol., 2015, 77, 24-35.

3 N. K. Hong, Y. Hong, S. K. Min, M. K. Sun and K. Y. Suh, Effect of orientation and density of nanotopography in dermal wound healing, Biomaterials, 2012, 33, 8782-8792.

4 G. C. Gurtner, S. Werner, Y. Barrandon and M. T. Longaker, Wound repair and regeneration, Nature, 2008, 453, 314-321.

5 C. C. Yates, D. Whaley, R. Babu, J. Zhang, P. Krishna, E. Beckman, A. W. Pasculle and A. Wells, The effect of multifunctional polymer-based gels on wound healing in 
full thickness bacteria-contaminated mouse skin wound models, Biomaterials, 2007, 28, 3977-3986.

6 O. Ostvar, S. Shadvar, E. Yahaghi, K. Azma, A. F. Fayyaz, K. Ahmadi and I. Nowrouzian, Effect of platelet-rich plasma on the healing of cutaneous defects exposed to acute to chronic wounds: a clinico-histopathologic study in rabbits, Diagn. Pathol., 2015, 10, 1-6.

7 I. Iacopetti, A. Perazzi, V. Ferrari and R. Busetto, Application of Platelet-Rich Gel to Enhance Wound Healing in the Horse: A Case Report, J. Equine Vet. Sci., 2012, 32, 123-128.

8 P. A. M. Everts, Autologous Platelet-Leukocyte Enriched Gel. Basics and Efficacy, A novel method to support soft tissue and bone healing, Utrecht University, 2007.

9 L. J. Xian, S. R. Chowdhury, A. B. Saim and R. B. H. Idrus, Concentration-dependent effect of platelet-rich plasma on keratinocyte and fibroblast wound healing, Cytotherapy, 2014, 17, 293-300.

10 S. Kanokpanont, S. Damrongsakkul, J. Ratanavaraporn and P. Aramwit, An innovative bi-layered wound dressing made of silk and gelatin for accelerated wound healing, Int. J. Pharm., 2012, 436, 141-153.

11 J. Kundu, L. A. Poole-Warren, P. Martens and S. C. Kundu, Silk fibroin/poly(vinyl alcohol) photocrosslinked hydrogels for delivery of macromolecular drugs, Acta Biomater., 2012, 8, 1720-1729.

12 H. Yamada, Y. Igarashi, Y. Takasu, H. Saito and K. Tsubouchi, Identification of fibroin-derived peptides enhancing the proliferation of cultured human skin fibroblasts, Biomaterials, 2004, 25, 467-472.

13 A. Sugihara, K. Sugiura, H. Morita, T. Ninagawa, K. Tubouchi, R. Tobe, M. Izumiya, T. Horio, G. Abraham and S. Ikehara, Promotive Effects of a Silk Film on Epidermal Recovery from Full-Thickness Skin Wounds, Proc. Soc. Exp. Biol. Med., 2000, 225, 58-64.

14 G. Guan, L. Bai, B. Zuo, M. Li, Z. Wu, Y. Li and L. Wang, Promoted dermis healing from full-thickness skin defect by porous silk fibroin scaffolds (PSFSs), Bio-Med. Mater. Eng., 2010, 20, 295-308.

15 Z. Rui, L. Xiang, B. Sun, Z. Ying, D. Zhang, Z. Tang, X. Chen and C. Wang, Electrospun chitosan/sericin composite nanofibers with antibacterial property as potential wound dressings, Int. J. Biol. Macromol., 2014, 68, 92-97.

16 S. Zhaorigetu, M. Sasaki, H. Watanabe and N. Kato, Supplemental silk protein, sericin, suppresses colon tumorigenesis in 1,2-dimethylhydrazine-treated mice by reducing oxidative stress and cell proliferation, Biosci., Biotechnol., Biochem., 2001, 65, 2181-2186.

17 M. Sasaki, H. Yamada and N. Kato, Consumption of silk protein, sericin elevates intestinal absorption of zinc, iron, magnesium and calcium in rats, Nutr. Res., 2000, 20, 15051511.

18 N. Kato, S. Sato, A. Yamanaka, H. Yamada, N. Fuwa and M. Nomura, Silk protein, sericin, inhibits lipid peroxidation and tyrosinase activity, Biosci., Biotechnol., Biochem., 1998, 62, 145-147.

19 H. Li, L. Li, Y. Qian, K. Cai, Y. Lu, L. Zhong, W. Liu and L. Yang, Preparation and cytocompatibility study of poly (epsilon-caprolactone)/silk sericin nanofibrous scaffolds, $J$. Biomed. Eng., 2011, 28, 305-309.

20 D. H. Whitman, R. L. Berry and D. M. Green, Platelet gel: an autologous alternative to fibrin glue with applications in oral and maxillofacial surgery, J. Oral Maxillofac. Surg., 1997, 55, 1294-1299.

21 J. Choukroun, A. Diss, A. Simonpieri, M. O. Girard, C. Schoeffler, S. L. Dohan, A. J. J. Dohan, J. Mouhyi and D. M. Dohan, Platelet-rich fibrin (PRF): a secondgeneration platelet concentrate. Part V: histologic evaluations of PRF effects on bone allograft maturation in sinus lift, Oral Surgery, Oral Medicine, Oral Pathology, Oral Radiology, and Endodontology, 2006, 101, 299-303.

22 E. Anitua, I. Andia, B. Ardanza, P. Nurden and A. T. Nurden, Autologous platelets as a source of proteins for healing and tissue regeneration, Thromb. Haemostasis, 2004, 91, 4-15.

23 B. Lu, T. Wang, Z. Li, F. Dai, L. Lv, F. Tang, K. Yu, J. Liu and G. Lan, Healing of skin wounds with a chitosan-gelatin sponge loaded with tannins and platelet-rich plasma, Int. J. Biol. Macromol., 2016, 82, 884-891.

24 M. J. Carter, C. P. Fylling and L. K. Parnell, Use of platelet rich plasma gel on wound healing: a systematic review and meta-analysis, Eplasty, 2011, 11, e38.

25 N. Morimoto, K. Yoshimura, M. Niimi, T. Ito, R. Aya, J. Fujitaka, H. Tada, S. Teramukai, T. Murayama and C. Toyooka, Novel collagen/gelatin scaffold with sustained release of basic fibroblast growth factor: clinical trial for chronic skin ulcers, Tissue Eng., Part A, 2013, 19, 1931-1940.

26 R. Ito, N. Morimoto, L. H. Pham, T. Taira, K. Kawai and S. Suzuki, Efficacy of the controlled release of concentrated platelet lysate from a collagen/gelatin scaffold for dermislike tissue regeneration, Tissue Eng., Part A, 2013, 19, 13981405.

27 B. Lu, T. Wang, Z. Li, F. Dai, L. Lv, F. Tang, K. Yu, J. Liu and G. Lan, Healing of skin wounds with a chitosan-gelatin sponge loaded with tannins and platelet-rich plasma, Int. J. Biol. Macromol., 2016, 82, 884-891.

28 B. S. Anisha, D. Sankar, A. Mohandas, K. P. Chennazhi, S. V. Nair and R. Jayakumar, Chitosan-hyaluronan/nano chondroitin sulfate ternary composite sponges for medical use, Carbohydr. Polym., 2013, 92, 1470-1476.

29 G. Lan, B. Lu, T. Wang, L. Wang, J. Chen, K. Yu, J. Liu, F. Dai and $\mathrm{D}$. $\mathrm{Wu}$, Chitosan/gelatin composite sponge is an absorbable surgical hemostatic agent, Colloids Surf., B, 2015, 136, 1026-1034.

30 T. J. Wu, H. H. Huang, C. W. Lan, C. H. Lin, F. Y. Hsu and Y. J. Wang, Studies on the microspheres comprised of reconstituted collagen and hydroxyapatite, Biomaterials, 2004, 25, 651-658.

31 Z. Fan, B. Liu, J. Wang, S. Zhang, Q. Lin, P. Gong, L. Ma and S. Yang, A Novel Wound Dressing Based on Ag/Graphene Polymer Hydrogel: Effectively Kill Bacteria and Accelerate Wound Healing, Adv. Funct. Mater., 2014, 24, 3933-3943.

32 L. D. Bartolo, S. Morelli, A. Bader and E. Drioli, The influence of polymeric membrane surface free energy on cell metabolic functions, J. Mater. Sci.: Mater. Med., 2001, 12, 959-963. 
33 M. Lampin, R. Warocquier-Clérout, C. Legris, M. Degrange and M. F. Sigot-Luizard, Correlation between substratum roughness and wettability, cell adhesion, and cell migration, J. Biomed. Mater. Res., 1997, 36, 99-108.

34 R. Murugan and S. Ramakrishna, Nano-featured scaffolds for tissue engineering: a review of spinning methodologies, Tissue Eng., 2006, 12, 435-447.

35 T. Maver, U. Maver, F. Mostegel, T. Griesser, S. Spirk, D. M. Smrke and K. Stana-Kleinschek, Cellulose based thin films as a platform for drug release studies to mimick wound dressing materials, Cellulose, 2015, 22, 749-761.

36 T. Maver, S. Hribernik, T. Mohan, D. M. Smrke, U. Maver and K. Stana-Kleinschek, Functional Wound Dressing Materials with Highly Tunable Drug Release Properties, RSC Adv., 2015, 5, 77873-77884.

37 K. O. Kim, Y. Lee, J. W. Hwang, H. Kim, M. K. Sun, S. W. Chang, H. S. Lee and Y. S. Choi, Wound healing properties of a 3-D scaffold comprising soluble silkworm gland hydrolysate and human collagen, Colloids Surf., B, 2014, 116, 318-326.

38 A. Vasconcelos, A. C. Gomes and A. Cavaco-Paulo, Novel silk fibroin/elastin wound dressings, Acta Biomater., 2012, 8, 3049-3060.

39 D. Archana, L. Upadhyay, R. P. Tewari, J. Dutta, Y. B. Huang and P. K. Dutta, Chitosan-pectin-alginate as a novel scaffold for tissue engineering applications, Indian J. Biotechnol., 2013, 12, 475-482.

40 E. Sugimori, S. Shintani, K. Ishikawa and H. Hamakawa, Effects of apatite foam combined with platelet-rich plasma on regeneration of bone defects, Dent. Mater. J., 2006, 25, 591-596.

41 M. G. Roubelakis, O. Trohatou, A. Roubelakis, E. Mili, I. Kalaitzopoulos, G. Papazoglou, K. I. Pappa and N. P. Anagnou, Platelet-rich plasma (PRP) promotes fetal mesenchymal stem/stromal cell migration and wound healing process, Stem Cell Rev., 2014, 10, 417-428.

42 W. F. wolkers, N. F. Walkers and J. H. Crowe, Human Platelets Loaded with Trehalose Survive Freeze-Drying, Cryobiology, 2001, 42, 79-87.

43 G. Pietramaggiori, S. S. Scherer, J. C. Mathews, M. Alperovich, H. J. Yang, J. Neuwalder, J. M. Czeczuga,
R. H. Chan, C. T. Wagner and D. P. Orgill, Healing modulation induced by freeze-dried platelet-rich plasma and micronized allogenic dermis in a diabetic wound model, Wound Repair Regen., 2008, 16, 218-225.

44 M. H. Mohammadi, B. Molavi, S. Mohammadi, M. Nikbakht, A. M. Mohammadi, S. Mostafaei, A. H. Norooznezhad, A. A. Ghorbani and A. Ghavamzadeh, Evaluation of wound healing in diabetic foot ulcer using platelet-rich plasma gel: a single-arm clinical trial, Transfus. Apher. Sci., 2016, DOI: 10.1016/j.transci.2016.10.020.

45 J. S. Fernandez-Moure, J. L. V. Eps, F. J. Cabrera, Z. Barbosa, G. M. D. Rosal, B. K. Weiner, W. A. Ellsworth and E. Tasciotti, Platelet-rich plasma: a biomimetic approach to enhancement of surgical wound healing, J. Surg. Res., 2017, 207, 33-44.

46 D. G. Gilbertson, M. E. Duff, J. W. West, J. D. Kelly, P. O. Sheppard, P. D. Hofstrand, Z. Gao, K. Shoemaker, T. R. Bukowski and M. Moore, Platelet-derived Growth Factor C (PDGF-C), a Novel Growth Factor That Binds to PDGF $\alpha$ and $\beta$ Receptor, J. Biol. Chem., 2001, 276, 2740627414.

47 A. Younger, M. Penner and H. E. Montijo, Vancouver Experience of Recombinant Human Platelet-Derived Growth Factor, Foot Ankle Clin., 2016, 21, 771-776.

48 R. S. Dhillon, E. M. Schwarz and M. D. Maloney, Platelet-rich plasma therapy - future or trend?, Arthritis Res. Ther., 2012, 14, 1-10.

49 F. M. Young, W. Phungtamdet and B. J. S. Sanderson, Modification of MTT assay conditions to examine the cytotoxic effects of amitraz on the human lymphoblastoid cell line, WIL2NS, Toxicol. In Vitro, 2005, 19, 1051-1059.

50 R. Yamaguchi, H. Terashima, S. Yoneyama, S. Tadano and N. Ohkohchi, Effects of Platelet-Rich Plasma on Intestinal Anastomotic Healing in Rats: PRP Concentration is a Key Factor, J. Surg. Res., 2012, 173, 258-266.

51 Y. Liu, A. Kalén, O. Risto and O. Wahlström, Fibroblast proliferation due to exposure to a platelet concentrate in vitro is $\mathrm{pH}$ dependent, Wound Repair Regen., 2002, 10, 336340. 